\title{
The Laplacian Spread of Tricyclic Graphs *
}

\author{
Yanqing Chen ${ }^{1}$ and Ligong Wang ${ }^{2}{ }^{\dagger}$ \\ Department of Applied Mathematics, \\ Northwestern Polytechnical University, Xi'an, Shaanxi 710072, P. R. China. \\ ${ }^{1}$ yanqing_chen@126.com \\ ${ }^{2}$ ligongwangnpu@yahoo.com.cn
}

Submitted: Nov 28, 2008; Accepted: Jun 23, 2009; Published: Jul 2, 2009

Mathematics Subject Classifications: 05C50, $15 \mathrm{~A} 18$.

\begin{abstract}
The Laplacian spread of a graph is defined to be the difference between the largest eigenvalue and the second smallest eigenvalue of the Laplacian matrix of the graph. In this paper, we investigate Laplacian spread of graphs, and prove that there exist exactly five types of tricyclic graphs with maximum Laplacian spread among all tricyclic graphs of fixed order.
\end{abstract}

\section{Introduction}

In this paper, we consider only simple undirected graphs. Let $G=(V, E)$ be a graph with vertex set $V=V(G)=\left\{v_{1}, v_{2}, \ldots, v_{n}\right\}$ and edge set $E=E(G)$. The adjacency matrix of the graph $G$ is defined to be a matrix $A=A(G)=\left[a_{i j}\right]$ of order $n$, where $a_{i j}=1$ if $v_{i}$ is adjacent to $v_{j}$, and $a_{i j}=0$ otherwise. The spectrum of $G$ can be denoted by

$$
S(G)=\left(\lambda_{1}(G), \lambda_{2}(G), \ldots, \lambda_{n}(G)\right)
$$

where $\lambda_{1}(G) \geq \lambda_{2}(G) \geq \cdots \geq \lambda_{n}(G)$ are the eigenvalues of $A(G)$ arranged in weakly decreasing order. The spread of graph $G$ is defined as $\mathscr{S}_{A}(G)=\lambda_{1}(G)-\lambda_{n}(G)$. Generally, the spread of a square complex matrix $M$ is defined to be $s(M)=\max _{i, j}\left|\lambda_{i}-\lambda_{j}\right|$, where the maximum is taken over all pairs of eigenvalues of $M$. There have been some studies on the spread of an arbitrary matrix $[8,15,17,18]$.

Recently, the spread of a graph has received much attention. In [16], Petrović determines all minimal graphs whose spread do not exceed 4. In [6], Gregory, Hershkowitz and

\footnotetext{
*Supported by the National Natural Science Foundation of China (No.10871158), the Natural Science Basic Research Plan in Shaanxi Province of China (No.SJ08A01), and SRF for ROCS, SEM.

${ }^{\dagger}$ Corresponding author.
} 
Kirkland present some lower and upper bounds for the spread of a graph. They show that the path is the unique graph with minimum spread among connected graphs of given order. However, the graph(s) with maximum spread is still unknown, and some conjectures are presented in their paper. In [10], Li, Zhang and Zhou determine the unique graph with maximum spread among all unicyclic graphs with given order not less than 18, which is obtained from a star by adding an edge between two pendant vertices. In [11] Bolian Liu and Muhuo Liu obtain some new lower and upper bounds for the spread of a graph, which are some improvements of Gregory's bound on the spread for graphs with additional restrictions.

Here we consider another version of spread of a graph, i.e. the Laplacian spread of a graph, which is defined as follows. Let $G$ be a graph as above. The Laplacian matrix of the graph $G$ is $L(G)=D(G)-A(G)$, where $D(G)=\operatorname{diag}\left(d\left(v_{1}\right), d\left(v_{2}\right), \ldots, d\left(v_{n}\right)\right)$ denotes the diagonal matrix of vertex degrees of $G$, and $d(v)$ denotes the degree of the vertex $v$ of $G$. The Laplacian spectrum of $G$ can be denoted by

$$
S L(G)=\left(\mu_{1}(G), \mu_{2}(G), \ldots, \mu_{n}(G)\right),
$$

where $\mu_{1}(G) \geq \mu_{2}(G) \geq \cdots \geq \mu_{n}(G)$ are the eigenvalues of $L(G)$ arranged in weakly decreasing order. We define the Laplacian spread of the graph $G$ as $\mathscr{S}_{L}(G)=\mu_{1}(G)-$ $\mu_{n-1}(G)$. Note that in the definition we consider the largest eigenvalue and the second smallest eigenvalue, as the smallest eigenvalue always equals zero.

Recently, the Laplacian spread of a graph has also received much attention. Yizheng Fan et al. have shown that among all trees of fixed order, the star is the unique one with maximum Laplacian spread and the path is the unique one with the minimum Laplacian spread [5]; among all unicyclic graphs of fixed order, the unique unicyclic graph with maximum Laplacian spread is obtained from a star by adding an edge between two pendant vertices [2]; and among all bicyclic graphs of fixed order, the only two bicyclic graphs with maximum Laplacian spread are obtained from a star by adding two incident edges and by adding two nonincident edges between the pendant vertices of the star, respectively [4].

A tricyclic graph is a connected graph in which the number of edges equals the number of vertices plus two. In this paper, we study the Laplacian spread of tricyclic graphs and determine that there are only five types of tricyclic graphs with maximum Laplacian spread among all tricyclic graphs of fixed order.

\section{Preliminaries}

In this section, we first introduce some preliminaries, which are needed in the following proofs. Let $G$ be a graph and let $v$ be a vertex of $G$. The neighborhood of $v$ in $G$ is denoted by $N(v)$, i.e. $N(v)=\{w: w v \in E(G)\}$. Denote by $\Delta(G)$ the maximum degree of all vertices of a graph $G$.

Lemma 2.1 [1] Let $G$ be a connected graph of order $n \geq 2$. Then

$$
\mu_{1}(G) \leq n
$$


with equality if and only if the complement graph of $G$ is disconnected.

Lemma 2.2 [3] Let $G$ be a connected graph with vertex set $\left\{v_{1}, v_{2}, \ldots, v_{n}\right\}(n \geq 2)$. Then

$$
\mu_{1}(G) \leq \max \left\{d\left(v_{i}\right)+d\left(v_{j}\right)-\left|N\left(v_{i}\right) \cap N\left(v_{j}\right)\right|: v_{i} v_{j} \in E(G)\right\} .
$$

Lemma 2.3 [12] Let $G$ be a connected graph with vertex set $\left\{v_{1}, v_{2}, \ldots, v_{n}\right\}(n \geq 2)$. Then

$$
\mu_{1}(G) \leq \max \left\{d\left(v_{i}\right)+m\left(v_{i}\right): v_{i} \in V(G)\right\}
$$

where $m\left(v_{i}\right)=\frac{\sum_{v_{j} \in N\left(v_{i}\right)} d\left(v_{j}\right)}{d\left(v_{i}\right)}$, the average of the degrees of the vertices adjacent to $v_{i}$.

Lemma 2.4 [7] Let $G$ be a graph of order $n \geq 2$ containing at least one edge. Then

$$
\mu_{1}(G) \geq \Delta(G)+1
$$

If $G$ is connected, then the equality holds if and only if $\Delta(G)=n-1$.

Lemma 2.5 [9] Let $G$ be a connected graph of order $n$ with a cutpoint $v$. Then $\mu_{n-1}(G) \leq$ 1 , with equality if and only if $v$ is adjacent to every vertex of $G$.

Lemma 2.6 Let $G$ be a connected graph of order $n \geq 3$ with two pendant vertices $u, v$ adjacent to a common vertex $w$. Then

$$
\mathscr{S}_{L}(G+u v) \leq \mathscr{S}_{L}(G)
$$

Proof. From the Corollary 3.9 of [13], we can get that 1 is in $S L(G)$ and $S L(G+u v)$ is $S L(G) \backslash\{1\} \cup\{3\}$. Since the largest eigenvalue in $S L(G)$ is at least $\triangle(G)+1 \geq 3$, the result follows.

\section{Main Results}

We introduce nineteen tricyclic graphs of order $n$ in Figure 1: the graphs $G_{1}(s ; n), s \geq 0$; $G_{2}(r, s ; n), r \geq 1, s \geq 0 ; G_{3}(r, s ; n), r \geq 0, s \geq 0 ; G_{4}(r, s ; n), r \geq 0, s \geq 0 ; G_{5}(r, s ; n)$, $s \geq r \geq 0 ; G_{6}(r, s ; n), r \geq 1, s \geq 1 ; G_{7}(r, s ; n), s \geq r \geq 1 ; G_{8}(r, s ; n), r \geq 0, s \geq 0$; $G_{9}(r, s ; n), r \geq 0, s \geq 0 ; G_{10}(r, s ; n), s \geq r \geq 0 ; G_{11}(r, s ; n), r \geq 0, s \geq 0 ; G_{12}(r, s ; n)$, $r \geq 0, s \geq 0 ; G_{13}(r, s ; n), r \geq 0, s \geq 0 ; G_{14}(r, s ; n), r \geq 1, s \geq 0 ; G_{15}(r, s ; n), s \geq r \geq 0$; $G_{16}(r, s ; n), s \geq r \geq 0 ; G_{17}(r, s ; n), s \geq r \geq 1 ; G_{18}(r, s ; n), s \geq r \geq 0 ; G_{19}(r, s ; n)$, $r \geq 0, s \geq 1$. Here $r, s$ are nonnegative integers, which are respectively the number of pendant vertices adjacent to some vertices of the related graphs.

Lemma 2.7 Let $G$ be any of the graphs $G_{1}(n-7 ; n), n \geq 7 ; G_{3}(0, n-6 ; n), n \geq 6$; $G_{4}(0, n-5 ; n), n \geq 6 ; G_{8}(0, n-5 ; n), n \geq 6$; and $G_{18}(0, n-4 ; n), n \geq 5$. Then

$$
\mathscr{S}_{L}(G)=n-1 \text {. }
$$

Proof. By Lemma 2.4 and Lemma 2.5, we can get the result easily. 


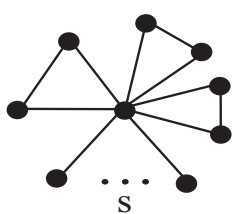

$G_{1}(s ; n)$

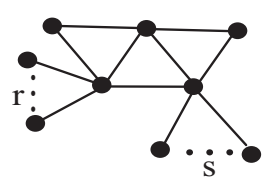

$G_{5}(r, s ; n)$

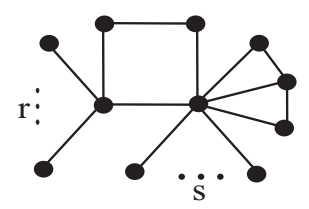

$G_{9}(r, s ; n)$

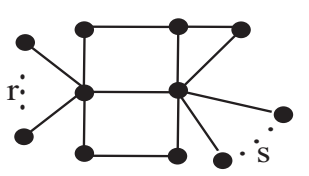

$G_{13}(r, s ; n)$

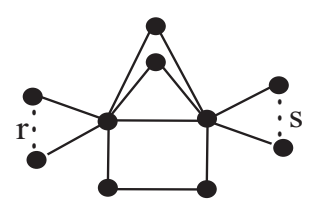

$G_{17}(r, s ; n)$

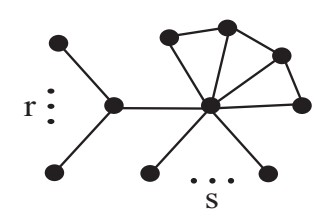

$G_{2}(r, s ; n)$

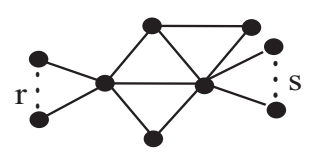

$G_{6}(r, s ; n)$

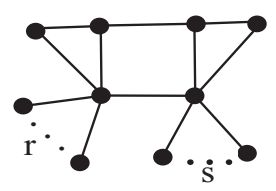

$G_{10}(r, s ; n)$

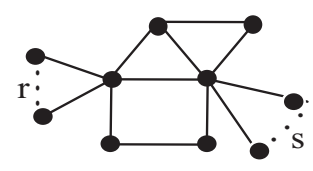

$G_{14}(r, s ; n)$

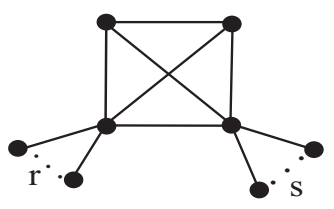

$G_{18}(r, s ; n)$
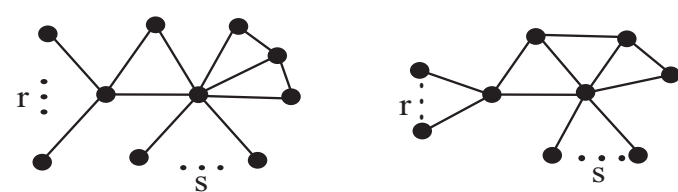

$G_{4}(r, s ; n)$

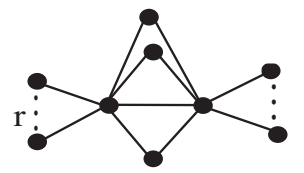

$G_{7}(r, s ; n)$

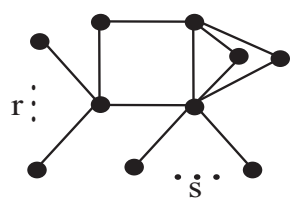

$G_{11}(r, s ; n)$

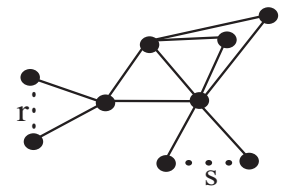

$G_{8}(r, s ; n)$

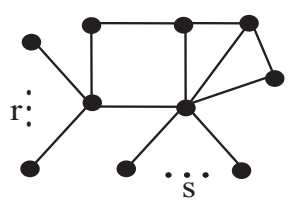

$G_{12}(r, s ; n)$

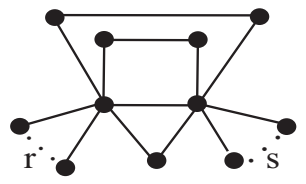

$G_{15}(r, s ; n)$

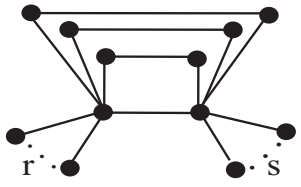

$G_{16}(r, s ; n)$

Figure 1: Nineteen tricyclic graphs on $n$ vertices.

In the following, we will prove that the graphs $G_{1}(n-7 ; n), n \geq 7 ; G_{3}(0, n-6 ; n), n \geq 6$; $G_{4}(0, n-5 ; n), n \geq 6 ; G_{8}(0, n-5 ; n), n \geq 6$; and $G_{18}(0, n-4 ; n), n \geq 4$ are the only tricyclic ones with maximum Laplacian spread. We first narrow down the possibility of the tricyclic graphs with maximum Laplacian spread.

Lemma 2.8 Let $G$ be a connected tricyclic graph with a triangle attached at a single vertex. Then $\mathscr{S}_{L}(G) \leq n-1$, the equality holds if and only if $G$ is $G_{1}(n-7 ; n), n \geq 7$ or $G_{3}(0, n-6 ; n), n \geq 6$.

Proof. Suppose that the graph $G$ has a triangle uvw attached at a single vertex $w$ (see Figure 2). By Lemma 2.6, $\mathscr{S}_{L}(G) \leq \mathscr{S}_{L}(G-u v)$. In addition, by Theorem 2.16 of [4] (that is, among all bicyclic graphs of fixed order, the only two bicyclic graphs with maximum Laplacian spread are obtained from a star by adding two incident edges and by adding two nonincident edges between the pendant vertices of the star, respectively), 
$\mathscr{S}_{L}(G-u v) \leq n-1$. Then $\mathscr{S}_{L}(G) \leq \mathscr{S}_{L}(G-u v) \leq n-1$. Moreover, if there exist such a graph $G$ with $\mathscr{S}_{L}(G)=n-1$, then $\mathscr{S}_{L}(G-u v)=n-1$ and so $G-u v$ (and consequently, $G$ ) must have a vertex of degree $n-1$ (again, by Theorem 2.16 of [4]). Furthermore, by Lemma 2.7, $\mathscr{S}_{L}\left(G_{1}(n-7 ; n)\right)=n-1, n \geq 7$ and $\mathscr{S}_{L}\left(G_{3}(0, n-6 ; n)\right)=n-1, n \geq 6$. The result follows.
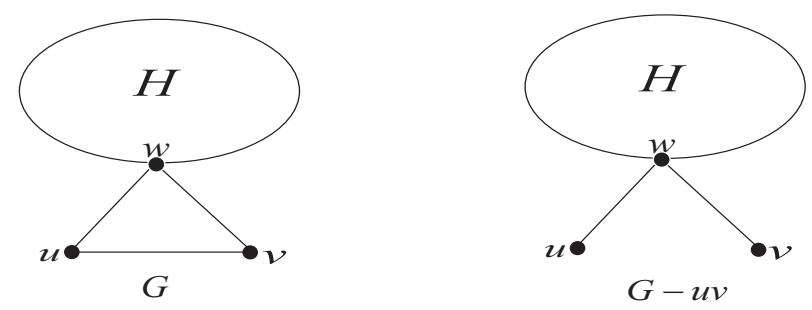

Figure 2

Lemma 2.9 Let $G$ be one with maximum Laplacian spread of all tricyclic graphs of order $n \geq 11$. Then $G$ is among the graphs $G_{1}(n-7 ; n), G_{2}(1, n-7 ; n), G_{3}(0, n-6 ; n)$, $G_{3}(1, n-7 ; n), G_{4}(0, n-5 ; n), G_{4}(1, n-6 ; n), G_{5}(0, n-5 ; n), G_{6}(1, n-6 ; n), G_{7}(1, n-$ $6 ; n), G_{8}(0, n-5 ; n), G_{8}(1, n-6 ; n), G_{9}(0, n-7 ; n), G_{11}(0, n-6 ; n), G_{12}(0, n-6 ; n)$, $G_{18}(0, n-4 ; n), G_{18}(1, n-5 ; n), G_{19}(n-6,1 ; n)$.

Proof. Let $v_{i} v_{j}$ be an edge of $G$. Then

$$
d\left(v_{i}\right)+d\left(v_{j}\right)-\left|N\left(v_{i}\right) \cap N\left(v_{j}\right)\right|=\left|N\left(v_{i}\right) \cup N\left(v_{j}\right)\right| \leq n,
$$

with equality holds if and only if $v_{i} v_{j}$ is adjacent to every vertex of $G$. Therefore, if $G$ has no edge that is adjacent to every vertex of $G$, then by Lemma $2.2, \mu_{1}(G) \leq n-1$ and hence $\mathscr{S}_{L}(G)=\mu_{1}(G)-\mu_{n-1}(G)<n-1$ as $\mu_{n-1}(G)>0$. In addition, if $G$ is a tricyclic graph with a triangle attached at a single vertex but not the graphs $G_{1}(n-7 ; n)$ and $G_{3}(0, n-6 ; n)$, then by Lemma $2.8, \mathscr{S}_{L}(G)<n-1$. However, by Lemma 2.7, $\mathscr{S}_{L}\left(G_{1}(n-7 ; n)\right)=\mathscr{S}_{L}\left(G_{3}(0, n-6 ; n)\right)=\mathscr{S}_{L}\left(G_{4}(0, n-5 ; n)\right)=\mathscr{S}_{L}\left(G_{8}(0, n-5 ; n)\right)=$ $\mathscr{S}_{L}\left(G_{18}(0, n-4 ; n)\right)=n-1$. So $G$ must be one graph in Figure 1 for some $r$ or $s$.

For the graph $G_{2}(r, s ; n)$ of Figure 1 with $1 \leq r \leq n-6,0 \leq s \leq n-7$, by Lemma 2.3,

$$
\mu_{1}\left(G_{2}(r, s ; n)\right) \leq \max \left\{r+1+\frac{n-1}{r+1}, s+5+\frac{n+5}{s+5}\right\} .
$$

For $n \geq 11, s \leq n-8$ and an arbitrary $r \geq 1$,

$$
\begin{aligned}
& r+1+\frac{n-1}{r+1} \leq \max \left\{2+\frac{n-1}{2}, n-5+\frac{n-1}{n-5}\right\} \leq n-1, \\
& s+5+\frac{n+5}{s+5} \leq \max \left\{5+\frac{n+5}{5}, n-3+\frac{n+5}{n-3}\right\} \leq n-1,
\end{aligned}
$$

and hence $\mu_{1}\left(G_{2}(r, s ; n)\right) \leq n-1, \mathscr{S}_{L}\left(G_{2}(r, s ; n)\right)<n-1$ as $\mu_{n-1}(G)>0$. 
For the graph $G_{3}(r, s ; n)$ of Figure 1 with $0 \leq r \leq n-6,0 \leq s \leq n-6$, by Lemma 2.3 ,

$$
\mu_{1}\left(G_{3}(r, s ; n)\right) \leq \max \left\{r+2+\frac{n+1}{r+2}, s+5+\frac{n+5}{s+5}\right\} .
$$

For $n \geq 11, s \leq n-8$ and an arbitrary $r$,

$$
\begin{aligned}
& r+2+\frac{n+1}{r+2} \leq \max \left\{2+\frac{n+1}{2}, n-4+\frac{n+1}{n-4}\right\} \leq n-1, \\
& s+5+\frac{n+5}{s+5} \leq \max \left\{5+\frac{n+5}{5}, n-3+\frac{n+5}{n-3}\right\} \leq n-1,
\end{aligned}
$$

and hence $\mu_{1}\left(G_{3}(r, s ; n)\right) \leq n-1, \mathscr{S}_{L}\left(G_{3}(r, s ; n)\right)<n-1$ as $\mu_{n-1}(G)>0$.

For the graph $G_{4}(r, s ; n)$ of Figure 1 with $0 \leq r \leq n-5,0 \leq s \leq n-5$, by Lemma 2.3

$$
\mu_{1}\left(G_{4}(r, s ; n)\right) \leq \max \left\{r+2+\frac{n+2}{r+2}, s+4+\frac{n+5}{s+4}\right\} .
$$

For $n \geq 11, s \leq n-7$ and an arbitrary $r$,

$$
\mu_{1}\left(G_{4}(r, s ; n)\right) \leq \max \left\{r+2+\frac{n+2}{r+2}, s+4+\frac{n+5}{s+4}\right\} \leq n-1 .
$$

and hence $\mu_{1}\left(G_{4}(r, s ; n)\right) \leq n-1, \mathscr{S}_{L}\left(G_{4}(r, s ; n)\right)<n-1$.

For the graph $G_{5}(r, s ; n)$ of Figure 1 with $0 \leq r \leq s \leq n-5$, by Lemma 2.3,

$$
\mu_{1}\left(G_{5}(r, s ; n)\right) \leq \max \left\{r+3+\frac{n+4}{r+3}, s+3+\frac{n+4}{s+3}\right\} .
$$

For $n \geq 10$ and $0 \leq r \leq s \leq n-6$,

$$
\mu_{1}\left(G_{5}(r, s ; n)\right) \leq \max \left\{r+3+\frac{n+4}{r+3}, s+3+\frac{n+4}{s+3}\right\} \leq n-1 .
$$

and hence $\mu_{1}\left(G_{5}(r, s ; n)\right) \leq n-1, \mathscr{S}_{L}\left(G_{5}(r, s ; n)\right)<n-1$.

For the graph $G_{6}(r, s ; n)$ of Figure $1, n \geq 11,1 \leq r \leq n-6$ and $1 \leq s \leq n-7$, by Lemma 2.3,

$$
\mu_{1}\left(G_{6}(r, s ; n)\right) \leq \max \left\{r+3+\frac{n+4}{r+3}, s+4+\frac{n+5}{s+4}\right\} \leq n-1 .
$$

and hence $\mu_{1}\left(G_{6}(r, s ; n)\right) \leq n-1, \mathscr{S}_{L}\left(G_{6}(r, s ; n)\right)<n-1$.

For the graph $G_{7}(r, s ; n)$ of Figure $1, n \geq 11$ and $1 \leq r \leq s \leq n-7$, by Lemma 2.3,

$$
\mu_{1}\left(G_{7}(r, s ; n)\right) \leq \max \left\{r+4+\frac{n+5}{r+4}, s+4+\frac{n+5}{s+4}\right\} \leq n-1 .
$$

and hence $\mu_{1}\left(G_{7}(r, s ; n)\right) \leq n-1, \mathscr{S}_{L}\left(G_{7}(r, s ; n)\right)<n-1$. 
For the graph $G_{8}(r, s ; n)$ of Figure $1, n \geq 11, s \leq n-7$ and an arbitrary $r$, by Lemma 2.3,

$$
\mu_{1}\left(G_{8}(r, s ; n)\right) \leq \max \left\{r+2+\frac{n+3}{r+2}, s+4+\frac{n+5}{s+4}\right\} \leq n-1 .
$$

and hence $\mu_{1}\left(G_{8}(r, s ; n)\right) \leq n-1, \mathscr{S}_{L}\left(G_{8}(r, s ; n)\right)<n-1$.

For the graph $G_{9}(r, s ; n)$ of Figure $1, n \geq 10, s \leq n-8$ and an arbitrary $r$, by Lemma 2.3,

$$
\mu_{1}\left(G_{9}(r, s ; n)\right) \leq \max \left\{r+2+\frac{n}{r+2}, s+5+\frac{n+4}{s+5}\right\} \leq n-1 .
$$

and hence $\mu_{1}\left(G_{9}(r, s ; n)\right) \leq n-1, \mathscr{S}_{L}\left(G_{9}(r, s ; n)\right)<n-1$.

For the graph $G_{10}(r, s ; n)$ of Figure $1, n \geq 8$ and arbitrary $r, s$, by Lemma 2.3,

$$
\mu_{1}\left(G_{10}(r, s ; n)\right) \leq \max \left\{r+3+\frac{n+2}{r+3}, s+3+\frac{n+2}{s+3}\right\} \leq n-1 .
$$

and hence $\mu_{1}\left(G_{10}(r, s ; n)\right) \leq n-1, \mathscr{S}_{L}\left(G_{10}(r, s ; n)\right)<n-1$.

For the graph $G_{11}(r, s ; n)$ of Figure $1, n \geq 10, s \leq n-7$ and an arbitrary $r$, by Lemma 2.3,

$$
\mu_{1}\left(G_{11}(r, s ; n)\right) \leq \max \left\{r+2+\frac{n}{r+2}, s+4+\frac{n+4}{s+4}\right\} \leq n-1 .
$$

and hence $\mu_{1}\left(G_{11}(r, s ; n)\right) \leq n-1, \mathscr{S}_{L}\left(G_{11}(r, s ; n)\right)<n-1$.

For the graph $G_{12}(r, s ; n)$ of Figure $1, n \geq 10, s \leq n-7$ and an arbitrary $r$, by Lemma 2.3,

$$
\mu_{1}\left(G_{12}(r, s ; n)\right) \leq \max \left\{r+2+\frac{n}{r+2}, s+4+\frac{n+4}{s+4}\right\} \leq n-1 .
$$

and hence $\mu_{1}\left(G_{12}(r, s ; n)\right) \leq n-1, \mathscr{S}_{L}\left(G_{12}(r, s ; n)\right)<n-1$.

For the graph $G_{13}(r, s ; n)$ of Figure $1, n \geq 9$ and arbitrary $r, s$, by Lemma 2.3,

$$
\mu_{1}\left(G_{13}(r, s ; n)\right) \leq \max \left\{r+3+\frac{n+1}{r+3}, s+4+\frac{n+3}{s+4}\right\} \leq n-1
$$

and hence $\mu_{1}\left(G_{13}(r, s ; n)\right) \leq n-1, \mathscr{S}_{L}\left(G_{13}(r, s ; n)\right)<n-1$.

For the graph $G_{14}(r, s ; n)$ of Figure $1, n \geq 10, s \leq n-7$ and an arbitrary $r \geq 1$, by Lemma 2.3,

$$
\mu_{1}\left(G_{14}(r, s ; n)\right) \leq \max \left\{r+3+\frac{n+3}{r+3}, s+4+\frac{n+4}{s+4}\right\} \leq n-1 .
$$

and hence $\mu_{1}\left(G_{14}(r, s ; n)\right) \leq n-1, \mathscr{S}_{L}\left(G_{14}(r, s ; n)\right)<n-1$.

For the graph $G_{15}(r, s ; n)$ of Figure $1, n \geq 9$ and arbitrary $r, s$, by Lemma 2.3,

$$
\mu_{1}\left(G_{15}(r, s ; n)\right) \leq \max \left\{r+4+\frac{n+3}{r+4}, s+4+\frac{n+3}{s+4}\right\} \leq n-1 .
$$


and hence $\mu_{1}\left(G_{15}(r, s ; n)\right) \leq n-1, \mathscr{S}_{L}\left(G_{15}(r, s ; n)\right)<n-1$.

For the graph $G_{16}(r, s ; n)$ of Figure $1, n \geq 8$ and arbitrary $r, s$, by Lemma 2.3,

$$
\mu_{1}\left(G_{16}(r, s ; n)\right) \leq \max \left\{r+4+\frac{n+2}{r+4}, s+4+\frac{n+2}{s+4}\right\} \leq n-1 .
$$

and hence $\mu_{1}\left(G_{16}(r, s ; n)\right) \leq n-1, \mathscr{S}_{L}\left(G_{16}(r, s ; n)\right)<n-1$.

For the graph $G_{17}(r, s ; n)$ of Figure $1, n \geq 10$ and $1 \leq r \leq s$, by Lemma 2.3,

$$
\mu_{1}\left(G_{17}(r, s ; n)\right) \leq \max \left\{r+4+\frac{n+4}{r+4}, s+4+\frac{n+4}{s+4}\right\} \leq n-1 .
$$

and hence $\mu_{1}\left(G_{17}(r, s ; n)\right) \leq n-1, \mathscr{S}_{L}\left(G_{17}(r, s ; n)\right)<n-1$.

For the graph $G_{18}(r, s ; n)$ of Figure $1, n \geq 11$ and $0 \leq r \leq s \leq n-6$, by Lemma 2.3,

$$
\mu_{1}\left(G_{18}(r, s ; n)\right) \leq \max \left\{r+3+\frac{n+5}{r+3}, s+3+\frac{n+5}{s+3}\right\} \leq n-1 .
$$

and hence $\mu_{1}\left(G_{18}(r, s ; n)\right) \leq n-1, \mathscr{S}_{L}\left(G_{18}(r, s ; n)\right)<n-1$.

For the graph $G_{19}(r, s ; n)$ of Figure $1, n \geq 11, r \leq n-7$ and an arbitrary $s \geq 1$, by Lemma 2.3,

$$
\mu_{1}\left(G_{19}(r, s ; n)\right) \leq \max \left\{r+4+\frac{n+5}{r+4}, s+1+\frac{n-1}{s+1}\right\} \leq n-1 .
$$

and hence $\mu_{1}\left(G_{19}(r, s ; n)\right) \leq n-1, \mathscr{S}_{L}\left(G_{19}(r, s ; n)\right)<n-1$.

By the above discussion, if $G$ is one with maximum Laplacian spread of all tricyclic graphs of order $n \geq 11$, then $G$ is among the graphs $G_{1}(n-7 ; n), G_{2}(1, n-7 ; n), G_{3}(0, n-$ $6 ; n), G_{3}(1, n-7 ; n), G_{4}(0, n-5 ; n), G_{4}(1, n-6 ; n), G_{5}(0, n-5 ; n), G_{6}(1, n-6 ; n)$, $G_{7}(1, n-6 ; n), G_{8}(0, n-5 ; n), G_{8}(1, n-6 ; n), G_{9}(0, n-7 ; n), G_{11}(0, n-6 ; n), G_{12}(0, n-6 ; n)$, $G_{18}(0, n-4 ; n), G_{18}(1, n-5 ; n), G_{19}(n-6,1 ; n)$. The result follows.

We next show that except the graphs $G_{1}(n-7 ; n), G_{3}(0, n-6 ; n), G_{4}(0, n-5 ; n)$, $G_{8}(0, n-5 ; n)$ and $G_{18}(0, n-4 ; n)$, the Laplacian spreads of the other graphs in Lemma 2.9 are all less than $n-1$ for a suitable $n$. Thus by a little computation for the graphs in Figure 1 of small order, $G_{1}(n-7 ; n), n \geq 7 ; G_{3}(0, n-6 ; n), n \geq 6 ; G_{4}(0, n-5 ; n), n \geq 6$; $G_{8}(0, n-5 ; n), n \geq 6$; and $G_{18}(0, n-4 ; n), n \geq 4$ are proved to be the only tricyclic graphs with maximum Laplacian spread among all tricyclic graphs of fixed order $n$.

In the following Lemmas 2.10-2.21, for convenience we simply write $\mu_{1}\left(G_{i}(r, s ; n)\right)$, $\mu_{n-1}\left(G_{i}(r, s ; n)\right)$ as $\mu_{1}, \mu_{n-1}$ respectively under no confusions.

Lemma 2.10 For $n \geq 7$

$$
\mathscr{S}_{L}\left(G_{2}(1, n-7 ; n)\right)<n-1 \text {. }
$$


Proof. The characteristic polynomial $\operatorname{det}\left(\lambda I-L\left(G_{2}(1, n-7 ; n)\right)\right)$ of $L\left(G_{2}(1, n-7 ; n)\right)$ is

$$
\lambda(\lambda-3)\left(\lambda^{2}-6 \lambda+7\right)(\lambda-1)^{n-7}\left[\lambda^{3}-(n+2) \lambda^{2}+(3 n-2) \lambda-n\right] .
$$

By Lemma 2.1 and Lemma $2.4, n>\mu_{1}>n-1 \geq 6$, and by Lemma $2.5, \mu_{n-1}<1$. So $\mu_{1}, \mu_{n-1}$ are both roots of the following polynomial:

$$
f_{1}(\lambda)=\lambda^{3}-(n+2) \lambda^{2}+(3 n-2) \lambda-n .
$$

Observe that

$$
(n-1)-\mathscr{S}_{L}\left(G_{2}(1, n-7 ; n)\right)=(n-1)-\left(\mu_{1}-\mu_{n-1}\right)=\left(n-\mu_{1}\right)-\left(1-\mu_{n-1}\right) .
$$

If we can show $n-\mu_{1}>1-\mu_{n-1}$, the result will follow. By Lagrange Mean Value Theorem,

$$
f_{1}(n)-f_{1}\left(\mu_{1}\right)=\left(n-\mu_{1}\right) f_{1}^{\prime}\left(\xi_{1}\right)
$$

for some $\xi_{1} \in\left(\mu_{1}, n\right)$. As $f_{1}^{\prime}(x)$ is positive and strict increasing on the interval $\left(\mu_{1}, n\right]$,

$$
n-\mu_{1}=\frac{f_{1}(n)-f_{1}\left(\mu_{1}\right)}{f_{1}^{\prime}\left(\xi_{1}\right)}>\frac{n^{2}-3 n}{f_{1}^{\prime}(n)}=1-\frac{2 n-2}{n^{2}-n-2},
$$

Note that the function $g_{1}(x)=\frac{2 x-2}{x^{2}-x-2}$ is strictly decreasing for $x \geq 7$. Hence

$$
\left(n-\mu_{1}\right)-\left(1-\mu_{n-1}\right)>\mu_{n-1}-g_{1}(n) \geq \mu_{n-1}-g_{1}(7)=\mu_{n-1}-0.3 .
$$

Observe that a star of order $n$ has eigenvalues: $0, n, 1$ of multiplicity $n-2$, and hence has $n-1$ eigenvalues not less than 1 . As $G_{2}(1, n-7 ; n)$ contains a star of order $n-1$, by eigenvalues interlacing theorem (that is, $\mu_{i}(G) \geq \mu_{i}(G-e)$ for $i=1,2, \ldots, n$ if we delete an edge $e$ from a graph $G$ of order $n$; or see [14]), $G_{2}(1, n-7 ; n)$ has $(n-2)$ eigenvalues not less than 1. Now $f_{1}(0.3)=-0.753-0.19 n<0$ and $f_{1}(1)=n-3>0$. So $0.3<\mu_{n-1}<1$. The result follows.

Lemma 2.11 For $n \geq 7$

$$
\mathscr{S}_{L}\left(G_{3}(1, n-7 ; n)\right)<n-1 .
$$

Proof. The characteristic polynomial $\operatorname{det}\left(\lambda I-L\left(G_{3}(1, n-7 ; n)\right)\right)$ of $L\left(G_{3}(1, n-7 ; n)\right)$ is

$$
\lambda(\lambda-2)(\lambda-4)(\lambda-1)^{n-7}\left[\lambda^{4}-(n+5) \lambda^{3}+(6 n+3) \lambda^{2}-(9 n-5) \lambda+3 n\right] .
$$

By Lemma 2.1 and Lemma 2.4, $n>\mu_{1}>n-1 \geq 6$, and by Lemma $2.5, \mu_{n-1}<1$. So $\mu_{1}, \mu_{n-1}$ are both roots of the following polynomial:

$$
f_{2}(\lambda)=\lambda^{4}-(n+5) \lambda^{3}+(6 n+3) \lambda^{2}-(9 n-5) \lambda+3 n,
$$

By Lagrange Mean Value Theorem,

$$
n-\mu_{1}=\frac{f_{2}(n)-f_{2}\left(\mu_{1}\right)}{f_{2}^{\prime}\left(\xi_{1}\right)}>\frac{n^{3}-6 n^{2}+8 n}{f_{2}^{\prime}(n)}=\frac{n(n-2)(n-4)}{(n-1)\left(n^{2}-2 n-5\right)}>\frac{n-4}{n-1},
$$


for some $\xi_{1} \in\left(\mu_{1}, n\right)$. In addition, by Taylor's Theorem,

$$
f_{2}\left(\mu_{n-1}\right)=f_{2}(1)+f_{2}^{\prime}(1)\left(\mu_{n-1}-1\right)+\frac{f_{2}^{\prime \prime}\left(\xi_{2}\right)}{2 !}\left(\mu_{n-1}-1\right)^{2},
$$

for some $\xi_{2} \in\left(\mu_{n-1}, 1\right)$. As $f_{2}^{\prime}(1)=0$ and $f_{2}^{\prime \prime}(x)$ is positive and strict decreasing on the open interval $(0,1)$,

$$
\left(1-\mu_{n-1}\right)^{2}=\frac{2(n-4)}{f_{2}^{\prime \prime}\left(\xi_{2}\right)}<\frac{2(n-4)}{f_{2}^{\prime \prime}(1)}=\frac{n-4}{3(n-2)} .
$$

If $n \geq 7, \frac{n-4}{n-1}>\sqrt{\frac{n-4}{3(n-2)}}$, and hence $n-\mu_{1}>1-\mu_{n-1}$. The result follows.

Lemma 2.12 For $n \geq 9$

$$
\mathscr{S}_{L}\left(G_{4}(1, n-6 ; n)\right)<n-1 \text {. }
$$

Proof. The characteristic polynomial of $L\left(G_{4}(1, n-6 ; n)\right)$ is

$\lambda(\lambda-1)^{n-7}\left[\lambda^{6}-(n+11) \lambda^{5}+(12 n+40) \lambda^{4}-(52 n+48) \lambda^{3}+(99 n-10) \lambda^{2}-(80 n-34) \lambda+21 n\right]$.

So $\mu_{1}, \mu_{n-1}$ are both roots of the following polynomial:

$f_{3}(\lambda)=\lambda^{6}-(n+11) \lambda^{5}+(12 n+40) \lambda^{4}-(52 n+48) \lambda^{3}+(99 n-10) \lambda^{2}-(80 n-34) \lambda+21 n$,

The derivative

$$
f_{3}^{\prime}(\lambda)=6 \lambda^{5}-5(n+11) \lambda^{4}+4(12 n+40) \lambda^{3}-3(52 n+48)^{2}+2(99 n-10) \lambda-(80 n-34)
$$

and the second derivative

$$
f_{3}^{\prime \prime}(\lambda)=30 \lambda^{4}-20(n+11) \lambda^{3}+12(12 n+40) \lambda^{2}-6(52 n+48)+2(99 n-10)
$$

As $f_{3}^{\prime}(x)$ is positive and strict increasing on the interval $\left(\mu_{1}, n\right]$, By Lagrange Mean Value Theorem,

$$
\begin{aligned}
n-\mu_{1} & =\frac{f_{3}(n)-f_{3}\left(\mu_{1}\right)}{f_{3}^{\prime}\left(\xi_{1}\right)}>\frac{n^{5}-12 n^{4}+51 n^{3}-90 n^{2}+55 n}{f_{3}^{\prime}(n)} \\
& =1-\frac{5 n^{4}-47 n^{3}+144 n^{2}-155 n+34}{n^{5}-7 n^{4}+4 n^{3}+54 n^{2}-100 n+34} \\
& >1-\frac{5 n^{4}-47 n^{3}+144 n^{2}-151 n}{n^{5}-7 n^{4}+4 n^{3}+54 n^{2}-100 n} \\
& =1-\frac{5 n^{3}-47 n^{2}+144 n-151}{n^{4}-7 n^{3}+4 n^{2}+54 n-100},
\end{aligned}
$$

for some $\xi_{1} \in\left(\mu_{1}, n\right)$. Note that the function

$$
g_{2}(x)=\frac{5 x^{3}-47 x^{2}+144 x-151}{x^{4}-7 x^{3}+4 x^{2}+54 x-100}
$$


is strictly decreasing for $x \geq 9$. Hence

$$
\left(n-\mu_{1}\right)-\left(1-\mu_{n-1}\right)>\mu_{n-1}-g_{2}(n) \geq \mu_{n-1}-g_{2}(9)=\mu_{n-1}-0.4534 .
$$

By a similar discussion to those in the last paragraph of the proof of Lemma 2.10, as $f_{3}(0.4534) \approx 10.3743+0.7208 n>0$ and $f_{3}(1)=-n+6<0.0 .4534<\mu_{n-1}<1$. The result follows.

Lemma 2.13 For $n \geq 7$

$$
\mathscr{S}_{L}\left(G_{5}(0, n-5 ; n)\right)<n-1 .
$$

Proof. The characteristic polynomial of $L\left(G_{5}(0, n-5 ; n)\right)$ is

$$
\lambda(\lambda-1)^{n-6}\left[\lambda^{5}-(n+10) \lambda^{4}+(11 n+29) \lambda^{3}-(40 n+16) \lambda^{2}+(54 n-19) \lambda-21 n\right] .
$$

So $\mu_{1}, \mu_{n-1}$ are both roots of the following polynomial:

$$
f_{4}(\lambda)=\lambda^{5}-(n+10) \lambda^{4}+(11 n+29) \lambda^{3}-(40 n+16) \lambda^{2}+(54 n-19) \lambda-21 n,
$$

By Lagrange Mean Value Theorem,

$$
n-\mu_{1}=\frac{f_{4}(n)-f_{4}\left(\mu_{1}\right)}{f_{4}^{\prime}\left(\xi_{1}\right)}>\frac{n^{4}-11 n^{3}+38 n^{2}-40 n}{f_{4}^{\prime}(n)}=1-\frac{4 n^{3}-31 n^{2}+62 n-19}{n^{4}-7 n^{3}+7 n^{2}+22 n-19},
$$

for some $\xi_{1} \in\left(\mu_{1}, n\right)$. Note that the function

$$
g_{3}(x)=\frac{4 x^{3}-31 x^{2}+62 x-19}{x^{4}-7 x^{3}+7 x^{2}+22 x-19}
$$

is strictly decreasing for $x \geq 7$. Hence

$$
\left(n-\mu_{1}\right)-\left(1-\mu_{n-1}\right)>\mu_{n-1}-g_{3}(7)=\mu_{n-1}-0.5607 .
$$

As $f_{4}(0.5607) \approx-11.5044-1.4574 n<0$ and $f_{4}(1)=3 n-15>0, \mu_{n-1}>0.5607$. The result follows.

Lemma 2.14 For $n \geq 8$

$$
\mathscr{S}_{L}\left(G_{6}(1, n-6 ; n)\right)<n-1 \text {. }
$$

Proof. The characteristic polynomial of $L\left(G_{6}(1, n-6 ; n)\right)$ is $\lambda(\lambda-1)^{n-7}\left[\lambda^{6}-(n+11) \lambda^{5}+(12 n+39) \lambda^{4}-(51 n+45) \lambda^{3}+(95 n-9) \lambda^{2}-(77 n-31) \lambda+21 n\right]$.

So $\mu_{1}, \mu_{n-1}$ are both roots of the following polynomial:

$f_{5}(\lambda)=\lambda^{6}-(n+11) \lambda^{5}+(12 n+39) \lambda^{4}-(51 n+45) \lambda^{3}+(95 n-9) \lambda^{2}-(77 n-31) \lambda+21 n$, 
By Lagrange Mean Value Theorem,

$$
\begin{aligned}
n-\mu_{1}= & \frac{f_{5}(n)-f_{5}\left(\mu_{1}\right)}{f_{5}^{\prime}\left(\xi_{1}\right)}>\frac{n^{5}-12 n^{4}+50 n^{3}-86 n^{2}+52 n}{f_{5}^{\prime}(n)} \\
& =1-\frac{5 n^{4}-47 n^{3}+141 n^{2}-147 n+31}{n^{5}-7 n^{4}+3 n^{3}+55 n^{2}-95 n+31} \\
& >1-\frac{5 n^{4}-47 n^{3}+141 n^{2}-143 n}{n^{5}-7 n^{4}+3 n^{3}+55 n^{2}-95 n} \\
& =1-\frac{5 n^{3}-47 n^{2}+141 n-143}{n^{4}-7 n^{3}+3 n^{2}+55 n-95},
\end{aligned}
$$

for some $\xi_{1} \in\left(\mu_{1}, n\right)$. Note that the function

$$
g_{4}(x)=\frac{5 x^{3}-47 x^{2}+141 x-143}{x^{4}-7 x^{3}+3 x^{2}+55 x-95}
$$

is strictly decreasing for $x \geq 8$. Hence

$$
\left(n-\mu_{1}\right)-\left(1-\mu_{n-1}\right)>\mu_{n-1}-g_{4}(n) \geq \mu_{n-1}-g_{4}(8)=\mu_{n-1}-0.5119 .
$$

As $f_{5}(0.5119) \approx 9.7836+0.4254 n>0$ and $f_{5}(1)=-n+6<0, \mu_{n-1}>0.5119$. The result follows.

Lemma 2.15 For $n \geq 8$

$$
\mathscr{S}_{L}\left(G_{7}(1, n-6 ; n)\right)<n-1 .
$$

Proof. The characteristic polynomial of $L\left(G_{7}(1, n-6 ; n)\right)$ is

$$
\lambda(\lambda-2)^{2}(\lambda-1)^{n-7}\left[\lambda^{4}-(n+7) \lambda^{3}+(8 n+5) \lambda^{2}-(13 n-7) \lambda+5 n\right] .
$$

So $\mu_{1}, \mu_{n-1}$ are both roots of the following polynomial:

$$
f_{6}(\lambda)=\lambda^{4}-(n+7) \lambda^{3}+(8 n+5) \lambda^{2}-(13 n-7) \lambda+5 n,
$$

and

$$
n-\mu_{1}=\frac{f_{6}(n)-f_{6}\left(\mu_{1}\right)}{f_{6}^{\prime}\left(\xi_{1}\right)}>\frac{n^{3}-8 n^{2}+12 n}{f_{6}^{\prime}(n)}=\frac{n(n-2)(n-6)}{(n-1)\left(n^{2}-4 n-7\right)}>\frac{n-6}{n-1},
$$

for some $\xi_{1} \in\left(\mu_{1}, n\right)$. In addition,

$$
f_{6}\left(\mu_{n-1}\right)=f_{6}(1)+f_{6}^{\prime}(1)\left(\mu_{n-1}-1\right)+\frac{f_{6}^{\prime \prime}\left(\xi_{2}\right)}{2 !}\left(\mu_{n-1}-1\right)^{2},
$$

for some $\xi_{2} \in\left(\mu_{n-1}, 1\right)$. Noting $f_{6}^{\prime}(1)=0$,

$$
\left(1-\mu_{n-1}\right)^{2}<\frac{2(n-6)}{f_{6}^{\prime \prime}(1)}=\frac{n-6}{5(n-2)}
$$

If $n \geq 8, \frac{n-6}{n-1}>\sqrt{\frac{n-6}{5(n-2)}}$, and hence $n-\mu_{1}>1-\mu_{n-1}$. The result follows. 
Lemma 2.16 For $n \geq 8$

$$
\mathscr{S}_{L}\left(G_{8}(1, n-6 ; n)\right)<n-1 .
$$

Proof. The characteristic polynomial of $L\left(G_{8}(1, n-6 ; n)\right)$ is

$$
\lambda(\lambda-2)(\lambda-1)^{n-7}\left[\lambda^{5}-(n+9) \lambda^{4}+(10 n+21) \lambda^{3}-(31 n+3) \lambda^{2}+(33 n-16) \lambda-10 n\right] .
$$

So $\mu_{1}, \mu_{n-1}$ are both roots of the following polynomial:

$$
f_{7}(\lambda)=\lambda^{5}-(n+9) \lambda^{4}+(10 n+21) \lambda^{3}-(31 n+3) \lambda^{2}+(33 n-16) \lambda-10 n,
$$

and

$$
n-\mu_{1}=\frac{f_{7}(n)-f_{7}\left(\mu_{1}\right)}{f_{7}^{\prime}\left(\xi_{1}\right)}>\frac{n^{4}-10 n^{3}+30 n^{2}-26 n}{f_{7}^{\prime}(n)}=1-\frac{4 n^{3}-29 n^{2}+53 n-16}{n^{4}-6 n^{3}+n^{2}+27 n-16},
$$

for some $\xi_{1} \in\left(\mu_{1}, n\right)$. Note that the function

$$
g_{5}(x)=\frac{4 x^{3}-29 x^{2}+53 x-16}{x^{4}-6 x^{3}+x^{2}+27 x-16}
$$

is strictly decreasing for $x \geq 8$. Hence

$$
\left(n-\mu_{1}\right)-\left(1-\mu_{n-1}\right)>\mu_{n-1}-g_{5}(8)=\mu_{n-1}-0.4658 .
$$

As $f_{7}(0.4658) \approx-6.3831-0.3911 n<0$ and $f_{7}(1)=n-6>0, \mu_{n-1}>0.4658$. The result follows.

Lemma 2.17 For $n \geq 7$

$$
\mathscr{S}_{L}\left(G_{9}(0, n-7 ; n)\right)<n-1 .
$$

Proof. The characteristic polynomial of $L\left(G_{9}(0, n-7 ; n)\right)$ is

$$
\lambda(\lambda-4)(\lambda-2)^{2}(\lambda-1)^{n-7}\left[\lambda^{3}-(n+3) \lambda^{2}+(4 n-2) \lambda-2 n\right] .
$$

So $\mu_{1}, \mu_{n-1}$ are both roots of the following polynomial:

$$
f_{8}(\lambda)=\lambda^{3}-(n+3) \lambda^{2}+(4 n-2) \lambda-2 n,
$$

By Lagrange Mean Value Theorem,

$$
\begin{gathered}
n-\mu_{1}=\frac{f_{8}(n)-f_{8}\left(\mu_{1}\right)}{f_{8}^{\prime}\left(\xi_{1}\right)}=\frac{n(n-4)}{f_{8}^{\prime}\left(\xi_{1}\right)}, \\
1-\mu_{n-1}=\frac{f_{8}(1)-f_{8}\left(\mu_{n-1}\right)}{f_{8}^{\prime}\left(\xi_{2}\right)}=\frac{n-4}{f_{8}^{\prime}\left(\xi_{2}\right)},
\end{gathered}
$$

for some $\xi_{1} \in\left(\mu_{1}, n\right)$ and $\xi_{2} \in\left(\mu_{n-1}, 1\right)$. If we can show

$$
\frac{n}{f_{8}^{\prime}\left(\xi_{1}\right)}>\frac{1}{f_{8}^{\prime}\left(\xi_{2}\right)}
$$


the result will follow.

Note that $f_{8}^{\prime}(\lambda)=3 \lambda^{2}-2(n+3) \lambda+4 n-2$. As $f_{8}^{\prime}(\lambda)$ is positive and strictly decreasing on the interval $(0,1)$, and is positive and strictly increasing on the interval $\left(\mu_{1},+\infty\right)$,

$$
\begin{aligned}
& n f_{8}^{\prime}\left(\xi_{2}\right)>n f_{8}^{\prime}(1)=n(2 n-5) . \\
& f_{8}^{\prime}\left(\xi_{1}\right)<f_{8}^{\prime}(n)=n^{2}-2 n-2 .
\end{aligned}
$$

Then

$$
n f_{8}^{\prime}\left(\xi_{2}\right)-f_{8}^{\prime}\left(\xi_{1}\right)>n^{2}-3 n+2>0
$$

The result follows.

Lemma 2.18 For $n \geq 8$

$$
\mathscr{S}_{L}\left(G_{11}(0, n-6 ; n)\right)<n-1 \text {. }
$$

Proof. The characteristic polynomial of $L\left(G_{11}(0, n-6 ; n)\right)$ is

$$
\lambda(\lambda-2)(\lambda-1)^{n-7}\left[\lambda^{5}-(n+9) \lambda^{4}+(10 n+22) \lambda^{3}-(32 n+8) \lambda^{2}+(38 n-12) \lambda-14 n\right] .
$$

So $\mu_{1}, \mu_{n-1}$ are both roots of the following polynomial:

$$
f_{9}(\lambda)=\lambda^{5}-(n+9) \lambda^{4}+(10 n+22) \lambda^{3}-(32 n+8) \lambda^{2}+(38 n-12) \lambda-14 n,
$$

and

$$
n-\mu_{1}=\frac{f_{9}(n)-f_{9}\left(\mu_{1}\right)}{f_{9}^{\prime}\left(\xi_{1}\right)}>\frac{n^{4}-10 n^{3}+30 n^{2}-26 n}{f_{9}^{\prime}(n)}=1-\frac{4 n^{3}-28 n^{2}+48 n-12}{n^{4}-6 n^{3}+2 n^{2}+22 n-12},
$$

for some $\xi_{1} \in\left(\mu_{1}, n\right)$. Note that the function

$$
g_{6}(x)=\frac{4 x^{3}-28 x^{2}+48 x-12}{x^{4}-6 x^{3}+2 x^{2}+22 x-12}
$$

is strictly decreasing for $x \geq 8$. Hence

$$
\left(n-\mu_{1}\right)-\left(1-\mu_{n-1}\right)>\mu_{n-1}-g_{6}(8)=\mu_{n-1}-0.4772 .
$$

As $f_{9}(0.4772) \approx-5.5994-2.1186 n<0$ and $f_{9}(1)=n-6>0, \mu_{n-1}>0.4772$. The result follows.

Lemma 2.19 For $n \geq 8$

$$
\mathscr{S}_{L}\left(G_{12}(0, n-6 ; n)\right)<n-1 \text {. }
$$


Proof. The characteristic polynomial of $L\left(G_{12}(0, n-6 ; n)\right)$ is $\lambda(\lambda-1)^{n-7}\left[\lambda^{6}-(n+11) \lambda^{5}+(12 n+41) \lambda^{4}-(53 n+55) \lambda^{3}+(106 n+4) \lambda^{2}-(94 n-26) \lambda+29 n\right]$.

So $\mu_{1}, \mu_{n-1}$ are both roots of the following polynomial:

$f_{10}(\lambda)=\lambda^{6}-(n+11) \lambda^{5}+(12 n+41) \lambda^{4}-(53 n+55) \lambda^{3}+(106 n+4) \lambda^{2}-(94 n-26) \lambda+29 n$, and

$$
\begin{aligned}
n-\mu_{1}= & \frac{f_{10}(n)-f_{10}\left(\mu_{1}\right)}{f_{10}^{\prime}\left(\xi_{1}\right)}>\frac{n^{5}-12 n^{4}+51 n^{3}-90 n^{2}+55 n}{f_{10}^{\prime}(n)} \\
& =1-\frac{5 n^{4}-46 n^{3}+137 n^{2}-141 n+26}{n^{5}-7 n^{4}+5 n^{3}+47 n^{2}-86 n+26} \\
& >1-\frac{5 n^{3}-46 n^{2}+137 n-137}{n^{4}-7 n^{3}+5 n^{2}+47 n-86},
\end{aligned}
$$

for some $\xi_{1} \in\left(\mu_{1}, n\right)$. Note that the function

$$
g_{7}(x)=\frac{5 x^{3}-46 x^{2}+137 x-137}{x^{4}-7 x^{3}+5 x^{2}+47 x-86}
$$

is strictly decreasing for $x \geq 8$. Hence

$$
\left(n-\mu_{1}\right)-\left(1-\mu_{n-1}\right)>\mu_{n-1}-g_{7}(8)=\mu_{n-1}-0.5125 .
$$

As $f_{10}(0.5125) \approx 9.4297+2.3247 n>0$ and $f_{10}(1)=-n+6<0, \mu_{n-1}>0.5125$. The result follows.

Lemma 2.20 For $n \geq 7$

$$
\mathscr{S}_{L}\left(G_{18}(1, n-5 ; n)\right)<n-1 \text {. }
$$

Proof. The characteristic polynomial of $L\left(G_{18}(1, n-5 ; n)\right)$ is

$$
\lambda(\lambda-4)(\lambda-1)^{n-6}\left[\lambda^{4}-(n+6) \lambda^{3}+(7 n+4) \lambda^{2}-(11 n-6) \lambda+4 n\right] .
$$

So $\mu_{1}, \mu_{n-1}$ are both roots of the following polynomial:

$$
f_{11}(\lambda)=\lambda^{4}-(n+6) \lambda^{3}+(7 n+4) \lambda^{2}-(11 n-6) \lambda+4 n,
$$

and

$$
n-\mu_{1}=\frac{f_{11}(n)-f_{11}\left(\mu_{1}\right)}{f_{11}^{\prime}\left(\xi_{1}\right)}>\frac{n^{3}-7 n^{2}+10 n}{f_{11}^{\prime}(n)}=\frac{n(n-2)(n-5)}{(n-1)\left(n^{2}-3 n-6\right)}>\frac{n-5}{n-1},
$$

for some $\xi_{1} \in\left(\mu_{1}, n\right)$. In addition,

$$
f_{11}\left(\mu_{n-1}\right)=f_{11}(1)+f_{11}^{\prime}(1)\left(\mu_{n-1}-1\right)+\frac{f_{11}^{\prime \prime}\left(\xi_{2}\right)}{2 !}\left(\mu_{n-1}-1\right)^{2}
$$


for some $\xi_{2} \in\left(\mu_{n-1}, 1\right)$. Noting $f_{11}^{\prime}(1)=0$,

$$
\left(1-\mu_{n-1}\right)^{2}=\frac{2(n-5)}{f_{11}^{\prime \prime}\left(\xi_{2}\right)}<\frac{2(n-5)}{f_{11}^{\prime \prime}(1)}=\frac{n-5}{4(n-2)} .
$$

If $n \geq 7, \frac{n-5}{n-1}>\sqrt{\frac{n-5}{4(n-2)}}$, and hence $n-\mu_{1}>1-\mu_{n-1}$. The result follows.

Lemma 2.21 For $n \geq 7$

$$
\mathscr{S}_{L}\left(G_{19}(n-6,1 ; n)\right)<n-1 .
$$

Proof. The characteristic polynomial of $L\left(G_{19}(n-6,1 ; n)\right)$ is

$$
\lambda(\lambda-4)^{2}(\lambda-1)^{n-6}\left[\lambda^{3}-(n+2) \lambda^{2}+(3 n-2) \lambda-n\right] .
$$

So $\mu_{1}, \mu_{n-1}$ are both roots of the following polynomial:

$$
f_{12}(\lambda)=\lambda^{3}-(n+2) \lambda^{2}+(3 n-2) \lambda-n .
$$

By a similar discussion of Lemma 2.10, the result follows.

From the previous discussion, we can get that $G_{1}(n-7 ; n), G_{3}(0, n-6 ; n), G_{4}(0, n-$ $5 ; n), G_{8}(0, n-5 ; n)$ and $G_{18}(0, n-4 ; n)$ of Figure 1 are the only five graphs with maximum Laplacian spread among all tricyclic graphs of order $n \geq 11$. Moreover, for $5 \leq n \leq 10$, if $G$ is one with maximum Laplacian spread of all tricyclic graphs of order $n$, then $G$ is necessary among the graphs in Figure 1 (by the first paragraph of the proof of Lemma 2.9 ), and we can determine that the Laplacian spreads of the graphs in Figure 1 are all less than $n-1$ (by Lemma 2.3 and Lemmas 2.10-2.21) except for the graphs shown in Figure 3. By a little computation (with Mathematica) for the graphs in Figure 3, we find that $G_{1}(n-7 ; n), 7 \leq n \leq 10 ; G_{3}(0, n-6 ; n), 6 \leq n \leq 10 ; G_{4}(0, n-5 ; n), 6 \leq n \leq 10$; $G_{8}(0, n-5 ; n), 6 \leq n \leq 10 ;$ and $G_{18}(0, n-4 ; n), 5 \leq n \leq 10$ of Figure 1 are the only graphs with maximum Laplacian spread among all tricyclic graphs of order $n$ for $5 \leq n \leq 10$.

Theorem $2.22 G_{1}(n-7 ; n), n \geq 7 ; G_{3}(0, n-6 ; n), n \geq 6 ; G_{4}(0, n-5 ; n), n \geq 6$; $G_{8}(0, n-5 ; n), n \geq 6$; and $G_{18}(0, n-4 ; n), n \geq 4$ of Figure 1 are the only graphs with maximum Laplacian spread among all tricyclic graphs of fixed order $n$. For each $n \geq 5$,

\begin{tabular}{|c|c|c|c|c|c|c|}
\hline \multicolumn{7}{|l|}{$\mathrm{n}=5$} \\
\hline graph & $G_{4}(0,0 ; 5)$ & $G_{7}(0,0 ; 5)$ & $G_{18}(0,1 ; 5)$ & & & \\
\hline spread & $2+\sqrt{2}$ & 3 & 4 & & & \\
\hline \multicolumn{7}{|l|}{$\mathrm{n}=6$} \\
\hline graph & $G_{3}(0,0 ; 6)$ & $G_{4}(0,1 ; 6)$ & $G_{4}(1,0 ; 6)$ & $G_{5}(0,1 ; 6)$ & $G_{8}(0,1 ; 6)$ & $G_{8}(1,0 ; 6)$ \\
\hline spread & 5 & 5 & 4.3871 & 4.4177 & 5 & $2 \sqrt{5}$ \\
\hline graph & $G_{10}(0,0 ; 6)$ & $G_{11}(0,0 ; 6)$ & $G_{12}(0,0 ; 6)$ & $G_{18}(0,2 ; 6)$ & $G_{18}(1,1 ; 6)$ & $G_{19}(0,1 ; 6)$ \\
\hline spread & 4 & $\sqrt{3}+\sqrt{2}+1$ & 4.1696 & 5 & $2 \sqrt{5}$ & 4.6002 \\
\hline
\end{tabular}
the maximum Laplacian spread is equal to $n-1$.

Remark There is only one tricyclic graph of order $n \leq 4$. It is $G_{18}(0,0 ; 4)=K_{4}$ with Laplacian spread 0. 


\begin{tabular}{|c|c|c|c|c|c|c|c|}
\hline \multicolumn{8}{|l|}{$\mathrm{n}=7$} \\
\hline graph & $G_{1}(0 ; 7)$ & $G_{3}(0,1 ; 7)$ & $G_{4}(0,2 ; 7)$ & $G_{4}(1,1 ; 7)$ & $G_{4}(2,0 ; 7)$ & $G_{5}(1,1 ; 7)$ & $G_{6}(1,1 ; 7)$ \\
\hline spread & 6 & 6 & 6 & 5.3905 & 4.8682 & 4.7921 & 5.3141 \\
\hline graph & $G_{7}(1,1 ; 7)$ & $G_{8}(0,2 ; 7)$ & $G_{8}(1,1 ; 7)$ & $G_{8}(2,0 ; 7)$ & $G_{10}(0,1 ; 7)$ & $G_{11}(0,1 ; 7)$ & $G_{11}(1,0 ; 7)$ \\
\hline spread & 5.3852 & 6 & 5.3716 & 5.0047 & 4.7399 & 5.2548 & 4.8846 \\
\hline graph & $G_{12}(0,1 ; 7)$ & $G_{12}(1,0 ; 7)$ & $G_{13}(0,0 ; 7)$ & $G_{14}(1,0 ; 7)$ & $G_{15}(0,0 ; 7)$ & $G_{18}(0,3 ; 7)$ & $G_{19}(0,2 ; 7)$ \\
\hline spread & 5.2536 & 4.7995 & 4.6031 & 4.7953 & $3+\sqrt{3}$ & 6 & 4.8635 \\
\hline \multicolumn{8}{|l|}{$\mathrm{n}=8$} \\
\hline graph & $G_{1}(1 ; 8)$ & $G_{2}(2,0 ; 8)$ & $G_{3}(0,2 ; 8)$ & $G_{3}(2,0 ; 8)$ & $G_{4}(0,3 ; 8)$ & $G_{4}(3,0 ; 8)$ & $G_{4}(1,2 ; 8)$ \\
\hline spread & 7 & 5.7675 & 7 & 5.6808 & 7 & 5.6589 & 6.4029 \\
\hline graph & $G_{4}(2,1 ; 8)$ & $G_{5}(1,2 ; 8)$ & $G_{6}(2,1 ; 8)$ & $G_{8}(0,3 ; 8)$ & $G_{8}(2,1 ; 8)$ & $G_{8}(3,0 ; 8)$ & $G_{9}(1,0 ; 8)$ \\
\hline spread & 5.6233 & 5.5986 & 5.7140 & 7 & 5.6194 & 5.7446 & 5.6824 \\
\hline graph & $G_{11}(1,1 ; 8)$ & $G_{12}(1,1 ; 8)$ & $G_{13}(0,1 ; 8)$ & $G_{14}(1,1 ; 8)$ & $G_{14}(2,0 ; 8)$ & $G_{15}(0,1 ; 8)$ & $G_{17}(1,1 ; 8)$ \\
\hline spread & 5.6472 & 5.6394 & 5.4620 & 5.5533 & 5.5595 & 5.4820 & 5.6811 \\
\hline graph & $G_{18}(2,2 ; 8)$ & $G_{19}(1,2 ; 8)$ & $G_{18}(0,4 ; 8)$ & $G_{19}(0,3 ; 8)$ & & & \\
\hline spread & $\sqrt{33}$ & 5.7675 & 7 & $2 \sqrt{7}$ & & & \\
\hline \multicolumn{8}{|l|}{$\mathrm{n}=9$} \\
\hline graph & $G_{1}(2 ; 9)$ & $G_{2}(2,1 ; 9)$ & $G_{3}(0,3 ; 9)$ & $G_{3}(2,1 ; 9)$ & $G_{4}(0,4 ; 9)$ & $G_{4}(2,2 ; 9)$ & $G_{5}(1,3 ; 9)$ \\
\hline spread & 8 & 6.7032 & 8 & 6.6231 & 8 & 6.5709 & 5.5375 \\
\hline graph & $G_{6}(2,2 ; 9)$ & $G_{6}(3,1 ; 9)$ & $G_{7}(2,2 ; 9)$ & $G_{8}(0,4 ; 9)$ & $G_{8}(2,2 ; 9)$ & $G_{9}(1,1 ; 9)$ & $G_{11}(1,2 ; 9)$ \\
\hline spread & 6.5144 & 6.5350 & 6.6332 & 8 & 6.5550 & 6.6219 & 6.5719 \\
\hline graph & $G_{12}(1,2 ; 9)$ & $G_{14}(1,2 ; 9)$ & $G_{17}(1,2 ; 9)$ & $G_{18}(0,5 ; 9)$ & $G_{18}(2,3 ; 9)$ & $G_{19}(2,2 ; 9)$ & \\
\hline spread & 6.5783 & 6.4790 & 6.4713 & 8 & 6.5553 & 6.7302 & \\
\hline \multicolumn{8}{|l|}{$\mathrm{n}=10$} \\
\hline graph & $G_{1}(3 ; 10)$ & $G_{2}(2,2 ; 10)$ & $G_{3}(0,4 ; 10)$ & $G_{3}(2,2 ; 10)$ & $G_{4}(0,5 ; 10)$ & $G_{4}(2,3 ; 10)$ & $G_{6}(2,3 ; 10)$ \\
\hline spread & 9 & 7.7142 & 9 & 7.6058 & 9 & 7.5591 & 7.4849 \\
\hline graph & $G_{7}(2,3 ; 10)$ & $G_{8}(0,5 ; 10)$ & $G_{8}(2,3 ; 10)$ & $G_{18}(0,6 ; 10)$ & $G_{18}(2,4$ & $G_{19}(3,2 ;$ & \\
\hline spread & 7.4654 & 9 & 7.5437 & 9 & 7.5212 & 6.7302 & \\
\hline
\end{tabular}

Figure 3 Laplacian spreads of some graphs of order $n$ in Figure 1 for $5 \leq n \leq 10$.

\section{Acknowledgements}

The authors are grateful to an anonymous referee for his helpful comments and suggestions. Particularly, he gives Lemma 2.6 and Lemma 2.8 that have helped to shorten the length of the paper.

\section{References}

[1] W. N. Anderson and T. D. Morely, Eigenvalues of the Laplacian of a graph, Linear Multilinear Algebra, 18(1985), 141-145.

[2] Y. Bao, Y. Tan, Y. Fan, The Laplacian spread of unicyclic graphs, Applied Mathematics Letters, (2009), In Press. 
[3] K. Das, An improved upper bound for Laplacian graph eigenvalues, Linear Algebra Appl., 368(2003), 269-278.

[4] Y. Fan, S. Li, Y. Tan, The Laplacian spread of bicyclic graphs, submitted.

[5] Y. Fan, J. Xu, Y. Wang, D. Liang, The Laplacian spread of a tree, Discrete Mathematics and Theoretical Computer Science, 10(1)(2008), 79-86.

[6] D. Gregory, D. Hershkowitz, S. Kirkland, The spread of the spectrum of a graph, Linear Algebra Appl., 332-334(2001), 23-35.

[7] R. Grone and R. Merris, The Laplacian spectrum of a graph II, SIAM J. Discrete Math., 7(1994), 229-237.

[8] C. R. Johnson, R. Kumar, H. Wolkowicz, Lower bounds for the spread of a matrix, Linear Algebra Appl., (71)1985, 161-173.

[9] S. Kirkland, A bound on the algebraic connectivity of a graph in terms of the number of cutpoints, Linear Multilinear Algebra, 47(2000), 93-103.

[10] X. Li, J. Zhang, B. Zhou, The spread of unicyclic graphs with given size of maximum matchings, Journal of Mathematical Chemistry, 42(4)(2007), 775-788.

[11] B. Liu, M. Liu, On the spread of the spectrum of a graph, Discrete Math, (2008) In Press.

[12] R. Merris, A note on Laplacian graph eigenvalues, Linear Algebra Appl., 285(1998), 33-35.

[13] R. Merris, Laplacian graph eigenvectors, Linear Algebra Appl., 278(1998), 221-236.

[14] B. Mohar, The Laplacian spectrum of graphs. In: Y. Alavi et al. (Eds.), Graph Theory, Combinatorics, and Applications, 1991, pp. 871-898. Wiley, New York.

[15] P. Nylen, T.Y. Tam, On the spread of a Hermitian matrix and a conjecture of Thompson, Linear Multilinear Algebra, (37)1994, 3-11.

[16] M. Petrović. On graphs whose spectral spread does not exceed 4, Publications de l'institut mathematique, 34(48)(1983), 169-174.

[17] R. C. Thompson, The eigenvalue spreads of a Hermitian matrix and its principal submatrices, Linear Multilinear Algebra, (32)1992, 327-333.

[18] X. Zhan, Extremal eigenvalues of real symmetric matrices with entries in an interval, SIAM. J. Matrix Anal. Appl., 27(2005), 851-860. 